\section{Counting the patient and healthcare organization costs of Clostridium difficile infection}

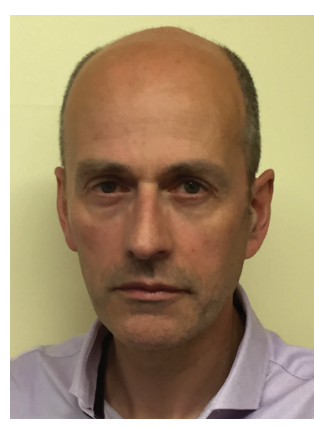

\begin{abstract}
Dr David Jenkins* is interviewed by Natasha Leeson, Commissioning Editor. David Jenkins is a consultant medical microbiologist and infection prevention and control (IPC) doctor at University Hospitals of Leicester (UHL) NHS Trust, a 1600-bed secondary/tertiary care healthcare organization in the center of England. He qualified as a medical doctor from King's College Hospital School of Medicine, University of London, in 1985 and undertook specialist training in medical microbiology at Brighton and Newcastle-upon-Tyne before taking up a consultant post in
\end{abstract} Leicester in 1997. He was responsible for his organization's response to the outbreak caused by the 027 ribotype of Clostridium difficile in 2006/2007 and for delivering annual reductions in C. difficile cases against nationally imposed targets, all of which have been met. He is also an honorary Senior Lecturer at the University of Leicester Medical School and Director of Healthcare Infection Prevention Limited, providing infection prevention consultancy services to healthcare organizations.

Q Why is Clostridium difficile such a serious healthcare issue?

Clostridium difficile infection (CDI) is a frequent cause of diarrheal disease in inpatients and is being increasingly recognized as a cause of diarrhea in primary care patients. At its worst, CDI can be fatal; one systematic review [1] of mortality associated with CDI reported that all-cause mortality at 30 days varied from 9 to $38 \%$, and attributable mortality at 30 days varied between 5.7 and $6.9 \%$. In-hospital mortality ranged from 8 to $37.2 \%$. The clinical features of CDI include prolonged episodes of painful, sometimes bloody, diarrhea, which can cause severe debilitation of patients. There is an appreciable risk, approximately $25 \%$, that infection will relapse, leading to one or more repeat episodes of diarrhea. CDI has significant healthcare resource implications. Inpatient length of stay is often prolonged. A study in four European countries [2] reported that CDI caused additional lengths of stay of 16 days in England, 15 days in Germany, 14 days in Spain and 13 days in the Netherlands. Such prolonged inpatient stay can have both financial and operational impacts on healthcare organizations. A study from Cambridge, England, in 1996 estimated the hospital cost of CDI to be over $£ 4000$ per episode [3]. As part of our seven-center study, the hospital cost of caring for patients with recurrent disease in 2014 was calculated and found to be over $£ 20,000$ per episode. Healthcare purchasers are increasingly unwilling to pay for costs incurred by complications of healthcare, such as CDI, so that the financial burden for managing these patients falls on the hospital concerned. Excessive bed occupancy caused by CDI can also limit the availability of beds, particularly during outbreaks when a large number of patients may be affected, and this can have additional healthcare and opportunity costs as the ability to admit patients from waiting lists is adversely affected and income associated with their admission is lost.

*University Hospitals of Leicester NHS Trust, Leicester LE1 5WW, UK; david.jenkins@uhl-tr.nhs.uk

\section{KEYWORDS}

- Clostridium difficile infection

- effectiveness studies $\bullet$ fidaxomicin

- healthcare resources $\bullet$ morbidity

- mortality • spores 
Q What effect did the emergence of ribotype 027 strains have on the number of cases?

In England, numbers of $C$. difficile cases reported voluntarily by laboratories increased from approximately 10,000 annually in 1996 to over 50,000 in 2007 when mandatory reporting was introduced. The majority of this increase was due to the emergence of the ribotype 027 strain, which caused a number of high-profile outbreaks in England around 2006/2007. In hospitals where ribotype 027 occurred, virtually all cases were due to the 027 ribotype. In addition, the nature of infection seemed to change to a much more aggressive illness; we saw an increase in the numbers of patients dying or undergoing colectomy, which seemed to be out of proportion to the increased number of infections.

\section{Q In the UK, how are C. difficile cases} identified, recorded \& monitored?

Because the organization of healthcare in the UK is a devolved function, each of the four regions of the UK - England, Scotland, Wales and Northern Ireland - address the management and surveillance of $C$. difficile in slightly different ways, which can make it difficult to compare rates of infection between the four regions. Furthermore, individual hospitals may interpret guidance on specimen collection and testing in different ways, which may account for variation in reports of $C$. difficile epidemiology. Ward staff should collect stool samples from any patients with diarrhea that is unexplained by other causes and submit these specimens to the laboratory for C. difficile testing. Laboratories should test for $C$. difficile in all fecal specimens that take the shape of their container - a standard description of a diarrhea specimen in UK microbiology laboratories - if the specimen comes from an inpatient or from a community patient age 65 years or older. As a minimum, the specimen should be tested using a two-stage test [4]. The first stage is a screening test using either a GDH enzyme immunoassay or a nucleic acid test for $C$. difficile toxin gene. The second stage confirmatory test should be a sensitive enzyme immunoassay for $C$. difficile toxin. Individual laboratories may wish to add a third stage test. Laboratories should then report all confirmed cases to the appropriate national body which, in England, is Public Health England. The national body will then report on epidemiological trends using denominators that, unfortunately, vary between the four
UK nations, making comparison between them very difficult.

\section{Q How important is it to correctly classify} patients based on severity or the potential for recurrence?

Assessing the severity of an infection can have important management consequences. It is critically important to promptly recognize patients with severe disease because they will need urgent specific treatment with either oral vancomycin or fidaxomicin, supportive care, including fluid management and, in very severe cases, colectomy. Identifying severe illness is especially important for patients whose onset of diarrhea occurs in the community, so that admission to hospital can be arranged as an emergency. Oral metronidazole or even no specific treatment may be appropriate for patients with moderate or mild infection. However, there is no general agreement about what constitutes mild, moderate or severe disease. Elderly patients, patients with a high white cell count or blood creatinine, fever or low albumin are likely to have severe disease. Identifying patients at risk of recurrence is even more challenging. Survival is a prerequisite for recurrence, so patients with mild disease may be more at risk of recurrence than patients with a severe first episode who do not survive this first attack. Having a local evidence-based protocol for the management of CDI in all its manifestations is an invaluable aid to delivering consistently high-quality treatment of this condition. The Public Health England publication 'Updated guidance on the management and treatment of Clostridium difficile infection' [5] is a highly recommended resource.

\section{Q What are current treatment options available for CDI?}

Specific treatment options are limited. Metronidazole is used for mild-to-moderate disease, but it is less effective than both vancomycin and fidaxomicin. These latter two agents have been shown by two randomized controlled Phase III trials to be of equal efficacy for the treatment of primary episodes of CDI [6,7]. However, these trials demonstrated a clear superiority of fidaxomicin over vancomycin for the prevention of recurrence of CDI. Patients treated with fidaxomicin had a risk of recurrence of between 12.7 and $15.4 \%$ versus 25.3 and $26.9 \%$ in vancomycin-treated patients. 
An alternative approach to the management of $\mathrm{CDI}$ is fecal microbiota transplant - instillation of a suspension of feces from a health volunteer by either a naso-gastric tube or through a colonscope. This is generally reserved for the management of patients whose disease is recurrent and resistant to more conventional treatments. It is supported by a randomized controlled trial [8] and has been adopted by some centers in the UK. However, given the non-specific nature of the donated feces, the potential risk of infection as a consequence of treatment and the possibility of transferring multidrug-resistant bacteria to the recipient, patients undergoing this treatment approach must be consented appropriately.

Q What can you tell me about your research? Randomized controlled trials aim to demonstrate that a treatment can work under ideal circumstances. However, they can be criticized because their selection criteria leads to the exclusion of many patients. Demonstrating that drugs work in a real-life setting, so-called effectiveness studies, is also important. My hospital, University Hospitals of Leicester (UK), was one of seven centers that introduced fidaxomicin for the treatment of CDI soon after it was licensed in the UK and which collaborated to analyze the effectiveness of this new drug in a routine clinical setting. The seven centers include district general hospitals as well as teaching hospitals and, therefore, represent a wide range of secondary care settings. In brief, the study was a before and after design. Treatment outcomes with metronidazole and/or vancomycin in the 12 months before the introduction of fidaxomicin were compared with outcomes in the subsequent 12 months when fidaxomicin was available for use. Centers varied in how they used fidaxomicin. Some used it as their sole agent for the treatment of CDI, while others used it alongside metronidazole or vancomycin, reserving it for more severely unwell patients or patients with recurrences. Overall, the results showed a reduction in recurrence rates following the introduction of fidaxomicin, from $11.7 \%$ prefidaxomcin to $8.8 \%$ after the introduction of fidaxomicin, with the greatest reduction, to $5.6 \%$, for those patients who were given it as first-line treatment for CDI. Two centers switched over to fidaxomicin as firstline treatment for all their CDI patients, and they saw a relative reduction in relapse rate of between 70 and $80 \%$. They also recorded significant reductions in 28 -day mortality rates.
Q What is the importance of the study's findings? Are there any limitations or potential challenges with this treatment option?

In my view, there are three important points to take away from this study. First, is the importance of performing effectiveness studies, to provide a reality check on the findings of Phase III randomized controlled trials. It turned out that the way fidaxomicin was used varied across the seven centers and there were corresponding differences in benefit seen as a result. I think it is very useful to see how variation from RCT protocols affects outcomes. Second, is that when fidaxomicin use replicated the approach used in the RCTs, the results were similar; sizeable reductions in relapse rates. Third, the effectiveness study approach allowed an economic analysis with local costs to be carried out. This showed that, despite significantly greater acquisition costs for fidaxomicin versus vancomycin, the lower recurrence rates associated with fidaxomicin use translate into overall cost savings as the direct management costs incurred by recurrences, predominately due to additional length of stay, are avoided through fidaxomicin use. Opportunity costs incurred when patients with recurrent disease occupy in-patient beds are also reduced as a consequence of fidaxomicin use.

\section{Q How important do you think alternative} treatment options, such as probiotics or intestinal microbiota transplantation, are for CDI?

While the use of fecal microbiota transplant is backed by RCT evidence, alternative approaches, such as probiotics and immunoglobulin, tend to suffer from less strong evidence to support their use. I prefer to use treatment strategies that are based on the foundations of sound evidence.

\section{Q How do you think we can prevent} C. difficile transmission from patients with CDI? The basis of patient-to-patient transmission of C. difficile is the spore of this organism. Spores present a great infection prevention challenge because of their resilience to heat, chemicals and dehydration. Our best chance of preventing transmission must be based on limiting environmental contamination with $C$. difficile spores. High quality environmental hygiene practices effective day-to-day cleaning, intensive cleaning and disinfection of single rooms following the discharge of CDI patients previously isolated in them, decontamination of commodes and 
other reusable medical devices - is absolutely essential. Good hand hygiene practice by staff and also by patients is equally as important. Preventing cases through antibiotic stewardship processes that limit antimicrobial prescribing courses and duration, especially of antibiotics such as cephalosporins and quinolones, should also be part of the strategy. In principle, contamination should reduce as cases decrease, which sets off a virtuous spiral of fewer cases and less contamination. A recently published study by Goldenberg and colleagues [9] reported a reduction in $C$. difficile spore environmental contamination when fidaxomicin was used for CDI treatment relative to metronidazole or vancomycin use, suggesting an additional benefit for fidaxomicin. If this effect is true and if it contributes to fewer cases, then fidaxomicin may have an action akin to the herd immunity proved by vaccines, protecting patients other than the recipient from disease.

\section{Q Where do you see this field of research going in the next 5 years?}

I think research in $C$. difficile and CDI will focus more and more on the $C$. difficile spore. The central role these objects play in the transmission and pathogenesis of CDI is its strength but also, potentially, its vulnerability. If we could identify ways to prevent spore formation, to prevent their outgrowth in patients or to encourage their outgrowth in the environment making the organism vulnerable to disinfectants, then we might stand a good chance of preventing transmission and infection.

\section{Financial \& competing interests disclosure}

$D$ Jenkins has received speaker and advisory board fees from Astellas (the manufacturer of fidaxomicin) and MSD. The seven centers study highlighted in the interview was financially supported by Astellas. D Jenkins has no other relevant affiliations or financial involvement with any organization or entity with a financial interest in or financial conflict with the subject matter or materials discussed in the manuscript apart from those disclosed.

No writing assistance was utilized in the production of this manuscript.

\section{Disclaimer}

The opinions expressed in this interview are those of the interviewee and do not necessarily reflect the views of Future Medicine Ltd.

\section{References}

1 Mitchell BG, Gardner A. Mortality and Clostridium diffcile infection: a review. Antimicrob. Resist. Infect. Control. 1, 20 (2012).

2 Eckmann C, Wasserman M, Latif F, Roberts G, Beriot-Mathiot A. Increased hospital length of stay attributable to Clostridium difficile infection in patients with four comorbidities: an analysis of hospital episode statistics in four European countries. Eur. J. Health Econ. 14, 835-846 (2013).

3 Wilcox MH, Cunniffe JG, Trundle C, Redpath C. Financial burden of hospital- acquired Clostridium difficile infection. J. Hosp. Infect. 34, 23-30 (1996).

4 Department of Health. Updated guidance on the diagnosis and report of Clostridium difficile (2012).

5 Public Health England. Updated guidance on the management and treatment of Clostridium difficile infection (2013).

6 Louie TJ, Miller MA, Mullane KM et al. Fidaxomicin versus vancomycin for Clostridium difficile infection. N. Engl. J. Med. 364, 422-431 (2011).

7 Cornely OA, Crook DW, Esposito R et al. Fidaxomicin versus vancomycin for infection with Clostridium difficile in Europe, Canada, and the USA: a double-blind, non-inferiority, randomised controlled trial. Lancet Infect. Dis. 12, 281-289 (2012).

8 Van Nood E, Vrieze A, Nieuwdorp M et al. Duodenal infusion of donor feces for recurrent Clostridium difficile. N. Engl. J. Med. 368, 407-415 (2013).

9 Biswas JS, Patel A, Otter JA et al. Reduction in Clostridium diffcile environmental contamination by hospitalized patients treated with fidaxomicin. J. Hosp. Infect. 90, 267-270 (2015). 